\title{
Dupilumab Provides Favorable Safety and Sustained Efficacy for up to 3 Years in an Open-Label Study of Adults with Moderate-to-Severe Atopic Dermatitis
}

\author{
Lisa A. Beck ${ }^{1} \cdot$ Diamant Thaçi $^{2} \cdot$ Mette Deleuran $^{3} \cdot$ Andrew Blauvelt $^{4} \cdot$ Robert Bissonnette $^{5}$. \\ Marjolein de Bruin-Weller ${ }^{6}$. Michihiro Hide ${ }^{7}$. Lawrence Sher ${ }^{8}$. Iftikhar Hussain ${ }^{9} \cdot$ Zhen Chen $^{10} \cdot$ Faisal A. Khokhar $^{10}$. \\ Bethany Beazley ${ }^{10} \cdot$ Marcella Ruddy $^{10} \cdot$ Naimish Patel $^{11} \cdot$ Neil M. H. Graham ${ }^{10} \cdot$ Marius Ardeleanu $^{10} \cdot$ Brad Shumel $^{10}$
}

Published online: 17 June 2020

(c) The Author(s) 2020

\begin{abstract} moderate-to-severe AD. The primary outcome was safety. $-65.4 \%$ ) at week 148 . dose. erate-to-severe $\mathrm{AD}$.

Trial Registration ClinicalTrials.gov: NCT01949311.

These data have been presented at the Las Vegas Dermatology Seminar 20th Annual Meeting (7 November 2019), the Pediatric Dermatology Research Alliance 2019 Annual Conference (14 November 2019), the Winter Clinical Dermatology Conference 2020 (17 January 2020), the MauiDerm for Dermatologists 16th Annual Meeting (25 January 2020), the Western Society of Allergy, Asthma and Immunology 58th Annual Meeting (2 February 2020), Deutschen Dermatologischen Gesellschaft Kompakt 2020 (7 February 2020), and the Hawaii Dermatology Seminar 44th Annual Meeting (16 February 2020).
\end{abstract}

Background Management of moderate-to-severe atopic dermatitis (AD) commonly requires long-term treatment.

Objective The aim of this study was to report the safety and efficacy of dupilumab treatment for up to 3 years in adults with

Methods This ongoing, multicenter, open-label extension study (LIBERTY AD OLE; NCT01949311) assessed dupilumab treatment in adults previously enrolled in dupilumab trials. Patients received dupilumab $300 \mathrm{mg}$ weekly up to 148 weeks.

Results Of 2677 patients enrolled and treated, 347 reached week 148. Mean self-reported drug compliance was $98.2 \%$. Safety data were consistent with previously reported trials (270.1 adverse events [AEs]/100 patient-years; 6.9 serious AEs/100 patient-years) and the known dupilumab safety profile. Common AEs ( $\geq 5 \%$ of patients) included nasopharyngitis, $A D$, upper respiratory tract infection, conjunctivitis, headache, oral herpes, and injection-site reactions. AD signs and symptoms showed sustained improvements during treatment with mean (standard deviation, mean percentage change from parent study baseline) Eczema Area and Severity Index 1.4 (3.2, - 95.4\%) and weekly Pruritus Numerical Rating Scale 2.2 (1.8,

Limitations No control arm; fewer patients at later time points; regimen different from the approved $300 \mathrm{mg}$ every 2 weeks

Conclusion These safety and efficacy results support dupilumab as a continuous long-term treatment for adults with mod-

Digital Features To view the digital features for this article go to: https://doi.org/10.6084/m9.figshare.12280337.

Electronic supplementary material The online version of this article (https://doi.org/10.1007/s40257-020-00527-x) contains supplementary material, which is available to authorized users.

\section{Key Points}

Dupilumab demonstrated favorable safety and sustained efficacy in adults with moderate-to-severe atopic dermatitis (AD) for up to 3 years.

The safety data reported in this open-label study are consistent with previously reported controlled studies of up to 52 weeks.

These safety and efficacy data support the long-term, continuous use of dupilumab in adults with moderate-tosevere AD.

Extended author information available on the last page of the article 


\section{Introduction}

Atopic dermatitis (AD) is a chronic inflammatory skin disease associated with eczematous lesions and pruritus that impairs quality of life [1] and often occurs with allergic comorbidities [1, 2]. Many systemic and topical treatments for $\mathrm{AD}$ are not recommended for long-term continuous use due to safety concerns and lack of long-term efficacy data [3].

Dupilumab is a fully human VelocImmune ${ }^{\circledR}$-derived $[4,5]$ monoclonal antibody that blocks the shared receptor subunit for interleukin (IL)-4 and IL-13. Dupilumab clinical trials have shown that these cytokines are key and central drivers of multiple type 2 inflammatory diseases [2, 6, 7]. Dupilumab is approved for patients with type 2 inflammatory diseases, including AD, asthma, and chronic rhinosinusitis with nasal polyps $[8,9]$. In multiple randomized, placebo-controlled phase III trials in patients with moderateto-severe AD, dupilumab with or without topical corticosteroids (TCSs) improved AD skin lesions, symptoms, and quality of life, and had a favorable safety profile [10-12]. Furthermore, in an open-label extension (OLE) study (LIBERTY AD OLE), up to 76 weeks of dupilumab treatment showed continued efficacy in AD signs, symptoms, and quality of life, with favorable safety [13]. In this study, we report the safety and efficacy of up to 3 years of dupilumab treatment in patients with moderate-to-severe AD from the LIBERTY AD OLE study.

\section{Methods}

\subsection{Study Design, Patients, and Treatment}

LIBERTY AD OLE is an ongoing, multicenter, openlabel trial in adults with moderate-to-severe AD (NCT01949311). The protocol is provided in electronic supplementary material 1 . The detailed study design and data (cut-off date April 2016) have been previously reported [13]. We report results with a cut-off date of 1 December 2018 (database lock 13 February 2019), at which time approximately 550 sites in 28 countries in North America, Europe, and Asia-Pacific had participated.

Patients were included if they participated in previous phase I-III dupilumab studies (including patients in the placebo groups) [10-12, 14-21] and adequately completed the required parent study assessments or were screened for phase III studies (NCT02277743/NCT02277769) [12], but not randomized due to randomization closure. Patients were ineligible if they had an adverse event (AE) deemed related to dupilumab that led to treatment discontinuation or had a serious AE deemed related to dupilumab in the parent study.

Patients enrolled from October 2013 received subcutaneous dupilumab $200 \mathrm{mg}$ weekly (400 mg loading dose). Following protocol amendment on 12 December 2013, patients received $300 \mathrm{mg}$ weekly based on the dose regimens selected for phase III studies.

Rescue medications included systemic corticosteroids (SCSs) and nonsteroidal systemic immunosuppressive medications (including phototherapy). Patients who received rescue medication discontinued study treatment for the duration of the rescue treatment plus five half-lives, after which they could resume dupilumab treatment. Protocol Amendment 7 allowed patients who used SCSs as rescue medication to continue treatment with study drug. Other concomitant treatments for AD, including TCSs and topical calcineurin inhibitors (TCIs), were permitted.

The original planned study duration per patient was up to 3 years of treatment or until regulatory approval/ commercial availability of dupilumab in the patient's geographic region, whichever came first. Country-specific protocol Amendment 7 for France, Germany, Poland, and Japan permitted those patients who had already completed 3 years of treatment to continue or restart and continue treatment through 31 December 2017. Region-specific Amendment 8 extended the treatment period to 5 years in Poland and Finland, and to September 2018 in France.

\subsection{Ethics}

The study was conducted following ethical principles that derive from the Declaration of Helsinki, the International Conference on Harmonisation guideline, Good Clinical Practice, and local applicable regulatory requirements, including Institutional Review Board approval. All patients provided written informed consent prior to undertaking any study procedures.

\subsection{Outcomes Assessed in This Analysis}

The primary outcome was incidence and rate (events per patient-year [PY]) of treatment-emergent AEs (TEAEs). Key secondary outcomes included incidence and rate of serious TEAEs, percentage of patients with an Investigator's Global Assessment (IGA) score of $0 / 1$, and percentage of patients with $\geq 75 \%$ improvement in the Eczema Area and Severity Index (EASI) from baseline (EASI-75). Other secondary outcomes were change and percentage change from baseline in EASI, percentage of patients with $\geq 50 \%$ or $\geq 90 \%$ improvement in EASI from baseline (EASI-50 and EASI90 , respectively), change and percentage change from baseline in the weekly average Pruritus Numerical Rating Scale 
(NRS), percentage of patients with $\geq 3$-point improvement (reduction) in weekly average Pruritus NRS from baseline or with a value of 0 , change from baseline in PatientOriented Eczema Measure (POEM) and Dermatology Life Quality Index (DLQI), and percentage of patients requiring rescue treatment. Post hoc outcomes included the percentage of patients with $\geq 2$-point improvement from baseline IGA score and percentage of patients with a weekly average Pruritus NRS score $\leq 3$ (mild itch) [22]. IGA and EASI assessments at the end of the treatment visit (week 148) were removed and subsequently restored from protocol Amendments 6 and 7, respectively.

\subsection{Statistical Analysis}

All analyses were performed in the safety analysis set (patients who received one or more doses of study drug). Duration-adjusted analyses for safety parameters were computed over the duration of study participation. Efficacy analyses are descriptive and were performed using all observed data at each time point, without any imputation for missing values; efficacy parameters were assessed up to week 148 . Sensitivity analyses on patients enrolled prior to December 2015 (to include only those enrolled 3 years prior to the data cut-off date of December 2018) were performed on selected key efficacy endpoints (mean and change from baseline of the parent study in EASI and weekly average Pruritus NRS) using multiple imputation and last observation carried forward methods. Because the OLE trial lacked a control arm, LIBERTY AD CHRONOS trial (NCT02260986) [10] safety results from the final analysis are provided as a comparison. CHRONOS was selected because it was the largest and longest (52 weeks) controlled study that required the use of concomitant TCS. All analyses were performed using SAS software version 9.4 (SAS Institute Inc., Cary, NC, USA).

\section{Results}

\subsection{Patients}

A total of 2826 patients were screened from 14 parent studies, of whom 2678 (94.8\%) were included in the OLE (Fig. 1). One screened patient was enrolled but not treated, leaving 2677 patients in the analysis set.

At database lock, $82.4 \%$ of patients (2207/2677) had completed up to week 52; 1028 (38.4\%) up to week 100; and $347(13.0 \%)$ up to week 148 (Table 1; Fig. 1). A total of 1061 (39.6\%) patients completed the study (treatment and safety follow-up); 291 (10.9\%) patients were receiving ongoing treatment; and 1325 (49.5\%) patients had withdrawn from the study (Table 1; Fig. 1). Most patients $(807,30.1 \%)$ withdrew from the study due to study termination by the sponsor upon regulatory approval/commercial availability of the study drug. 'Withdrawal by subject' accounted for 221 (8.3\%) withdrawals (see footnote, Table 1). Discontinuation due to AEs $(109,4.1 \%)$ and lack of efficacy $(57,2.1 \%)$ were infrequent. The mean self-reported study drug compliance was $98.2 \%$, with most patients ( $\geq 80 \%$ ) self-reporting injection compliance during the treatment period.

The majority of patients were male $(60.2 \%)$ and white (72.3\%); of the 147 enrolled patients who self-reported as black/African American, 134 (91.2\%) enrolled in the US, $9(6.1 \%)$ in Canada, $3(2.0 \%)$ in the UK, and $1(0.7 \%)$ in Austria; 541 patients self-reported as Asian, the majority from Japan (45.5\%), South Korea (17.7\%), US (15.5\%), and Canada (14.6\%) [Table 1]. A total of $62.7 \%$ of patients

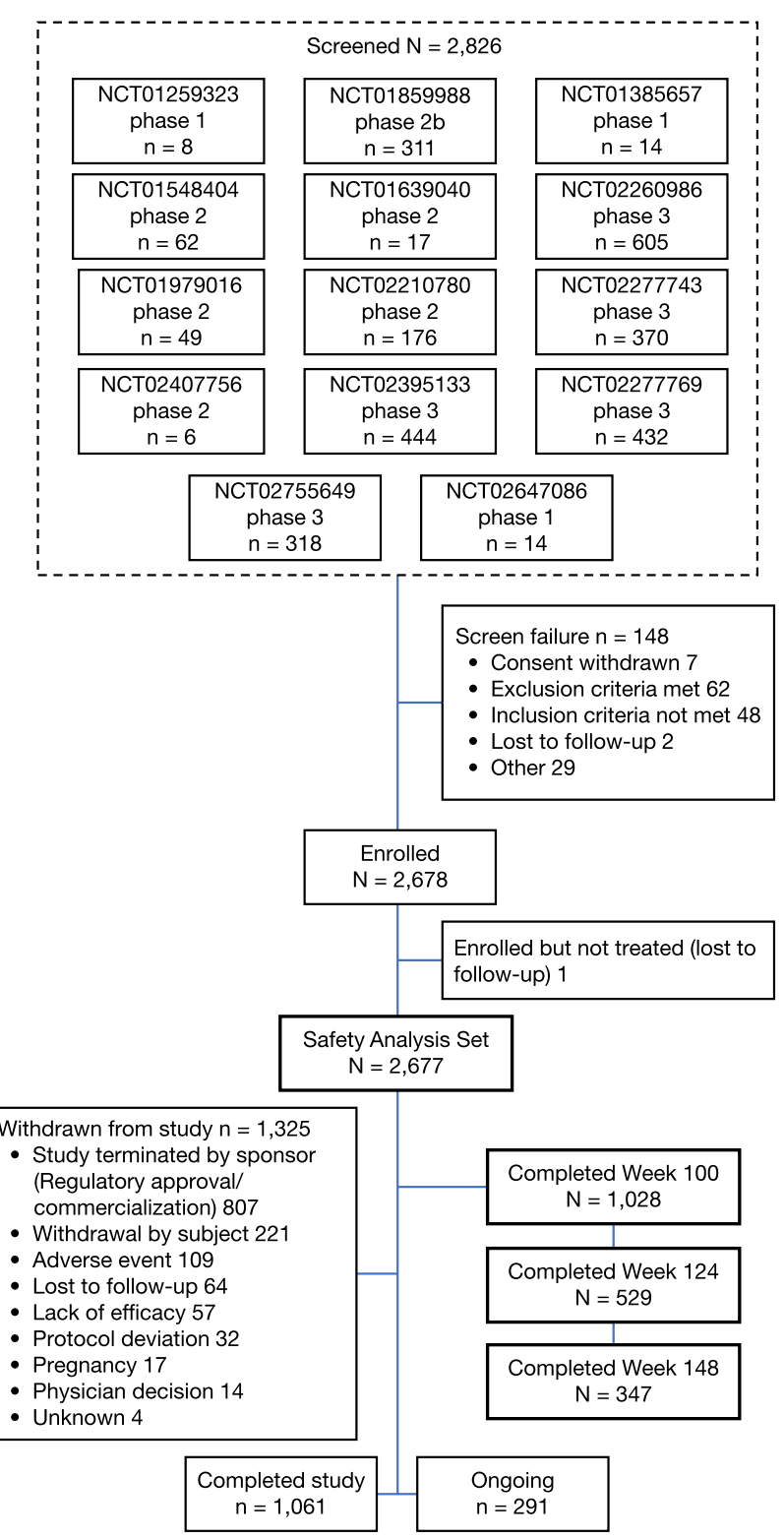

Fig. 1 Study flow diagram 
received dupilumab in the parent study, and $36.2 \%$ received placebo (Table 1). At baseline, almost all patients had one or more atopic/allergic comorbidities (Table 1). During the study, $52.9 \%$ and $16.1 \%$ of patients used TCSs and TCIs, respectively, with $55.5 \%$ of patients using TCSs or TCIs (Table 1).

\subsection{Safety Assessment}

Overall, 13,826 TEAEs were reported in 2264 patients (84.6\%), with an exposure-adjusted rate of 173.7 number of patients (nP)/100 PY (Table 2). Most TEAEs were of mildto-moderate severity; severe TEAEs were reported in $9.2 \%$ of patients. TEAEs led to treatment discontinuation in 95 patients (3.5\%). Two deaths were reported, both unrelated to study treatment (see footnote, Table 2).

The most common TEAEs were nasopharyngitis, AD, upper respiratory tract infection, conjunctivitis, headache, oral herpes, and injection-site reactions (Table 2), all previously reported in dupilumab trials. The exposure-adjusted incidence rates of these AEs were lower in the OLE compared with the 1-year CHRONOS trial (Table 2).

The exposure-adjusted incidence rate of conjunctivitis (reported as a cluster of Medical Dictionary for Regulatory Activities preferred terms, including conjunctivitis, conjunctivitis allergic, conjunctivitis bacterial, conjunctivitis viral, and atopic keratoconjunctivitis) was $11.96 \mathrm{nP} / 100 \mathrm{PY}$, comparable with that observed in the CHRONOS study placebo arm (9.24 nP/100 PY) and approximately 50\% lower than that observed in the $300 \mathrm{mg}$ weekly treatment arm of CHRONOS (23.37 nP/100 PY). Most conjunctivitis events were mild-to-moderate in severity and did not result in treatment discontinuation. In $>85 \%$ of cases, conjunctivitis resolved with standard ophthalmic treatments while the patient remained on study drug. The most commonly used ophthalmic treatments included intraocular corticosteroids, anti-allergy medications, and anti-infectives. Severe conjunctivitis occurred in $26(1.0 \%)$ patients. Fourteen $(0.5 \%)$ patients permanently discontinued the study drug due to conjunctivitis. The time to onset of all conjunctivitis events ranged from 9 to 574 days after the initiation of treatment in the OLE.

Rates of serious TEAEs and serious TEAEs considered by the investigator as related to treatment were similar between patients in the OLE and those receiving dupilumab in CHRONOS (Table 2). Overall, 354 serious TEAEs were reported in 256 patients $(9.6 \%)$. Thirty-six serious TEAEs considered related to study drug by the investigator occurred in $31(1.2 \%)$ patients, with an exposure-adjusted incidence rate of serious TEAEs in OLE $(0.61 \mathrm{nP} / 100 \mathrm{PY})$ lower than that observed in the placebo arm of CHRONOS $(1.06 \mathrm{nP} / 100 \mathrm{PY})$ and comparable with the dupilumab group $(0.68 \mathrm{nP} / 100 \mathrm{PY})$. Most serious treatment-related TEAEs occurred in individual patients. The most common serious TEAEs included osteoarthritis $(0.18 \mathrm{nP} / 100 \mathrm{PY})$, squamous cell carcinoma of the skin $(0.16 \mathrm{nP} / 100 \mathrm{PY})$, exacerbation of $\mathrm{AD}(0.12 \mathrm{nP} / 100 \mathrm{PY})$, syncope $(0.10 \mathrm{nP} / 100 \mathrm{PY})$, inguinal hernia $(0.10 \mathrm{nP} / 100 \mathrm{PY})$, and appendicitis $(0.10 \mathrm{nP} / 100 \mathrm{PY})$. Seven of nine patients with a serious TEAE of osteoarthritis had a medical history of this condition.

\subsection{Efficacy}

AD skin lesion severity and AD-related symptoms improved over the course of the OLE, characterized by rapid initial improvement followed by progressive incremental improvement thereafter. By week 100, 58.1\% of patients achieved IGA scores of 0 or 1 , increasing to $74.1 \%$ by week 148 (Table 3). By week 100, 77.2\% of patients achieved $\mathrm{a} \geq 2$-point improvement in IGA from baseline of the parent study, increasing to $87.9 \%$ at week 148 (Table 3).

Mean EASI at baseline of the parent study was 33.4, decreasing to 5.8 by week 16 of the OLE study and 1.5 by week 148 (observed cohort, Fig. 2). Mean weekly average Pruritus NRS at baseline of the parent study was 7.2, decreasing to 3.0 by week 10 and 2.1 by week 148 (observed cohort, Fig. 3). A sensitivity analysis on patients enrolled 3 years prior to the data cut-off date to assess any bias on treatment outcomes due to patient withdrawal showed EASI and weekly average Pruritus NRS results consistent with the observed data (Table 3; Figs. 2 and 3). Improvements in other EASI and Pruritus NRS assessments were sustained over the treatment period (Table 3). By week 148, 98.3\%, $96.6 \%$, and $87.9 \%$ of patients had achieved EASI-50/75/90, respectively, and $81.9 \%$ of patients had a weekly average Pruritus NRS $\leq 3$, with an absolute mean score of 2.2 (mild itch). By week 124, mean POEM had decreased from 20.5 at baseline of the parent study to 5.1, and mean DLQI from 14.7 to 2.9 (Table 3 ).

A total of 200 (7.5\%) patients received rescue medication, which was administered at the discretion of the investigator, and included SCSs $(187,7.0 \%)$ and/or non-steroidal immunosuppressants $(21,0.8 \%)$.

\section{Discussion}

In this analysis, dupilumab $300 \mathrm{mg}$ weekly (more frequent than the approved regimen of every other week) demonstrated a favorable safety profile and sustained efficacy in moderate-to-severe AD for up to 3 years. Safety data reported in this open-label study are consistent with controlled studies of up to 52 weeks [10-12, 22]. Data were consistent with the known dupilumab safety profile; nasopharyngitis, $\mathrm{AD}$, upper respiratory tract infection, conjunctivitis, 
Table 1 Study completion, reasons for withdrawal, and baseline demographics and disease characteristics

Dupilumab $300 \mathrm{mg}$ qw $[N=2677]^{\mathrm{a}}$

\section{OLE study completion status}

Completed up to 1 year

$2207(82.4)$

Completed up to 2 years

1028 (38.4)

Completed up to 3 years

347 (13.0)

Completed study ${ }^{\mathrm{b}}$

1061 (39.6)

Patients ongoing

291 (10.9)

Withdrawn from study

1325 (49.5)

Study terminated by sponsor (regulatory approval/commercialization)

$807(30.1)$

Withdrawal by subject ${ }^{\mathrm{c}}$

$221(8.3)$

Adverse event ${ }^{\mathrm{d}}$

$109(4.1)$

Lost to follow-up

$64(2.4)$

Lack of efficacy

$57(2.1)$

Protocol deviation

$32(1.2)$

Pregnancy

$17(0.6)$

Physician decision

$14(0.5)$

Unknown

$4(0.1)$

\section{Demographics at parent study baseline}

Age, years [mean (SD)]

$39.2(13.4)$

Male

$1611(60.2)$

Race

White

1936 (72.3)

Black or African American

147 (5.5)

Asian

$541(20.2)$

Other

33 (1.2)

Not reported

$20(0.7)$

$170.7(9.88)$

Height, cm [mean (SD)]

$77.1(18.4)$

Weight, kg [mean (SD)]

$26.4(5.59)$

BMI, $\mathrm{kg} / \mathrm{m}^{2}$ [mean (SD)]

$679(62.7)$

Previously treated with dupilumab

1679 (62.7)

Dupilumab 300 mg qw

$807(30.1)$

Dupilumab 300 mg q2w

$536(20.0)$

Other dupilumab doses ${ }^{\mathrm{e}}$

336 (12.6)

Received placebo qw in the parent study

968 (36.2)

Screen failure ${ }^{\mathrm{f}}$

$30(1.1)$

Patients with concurrent atopic/allergic disease history at parent study baseline

Allergic rhinitis

Asthma

Food allergy

$1010(37.7)$

Allergic conjunctivitis

Hives

$368(13.7)$

Chronic rhinosinusitis

Atopic keratoconjunctivitis

$78(2.9)$

Nasal polyps

$63(2.4)$

Eosinophilic esophagitis

$13(0.5)$

Concomitant topical treatments for AD during the study

Patients who used TCSs

1416 (52.9)

Patients who used TCIs 
Table 1 (continued)

\begin{tabular}{lll}
\hline & Dupilumab $300 \mathrm{mg}$ qw $[N=2677]^{\mathrm{a}}$ \\
\hline Patients who used TCSs or TCIs & $1487(55.5)$ & Parent study \\
Patients who used TCSs and TCIs & $360(13.4)$ & $29.0(14.8)$ \\
Disease characteristics & Current study (OLE) \\
Duration of AD, years [mean (SD)] & $29.9(14.8)$ & $32.8(13.2)$ \\
EASI (0-72) [mean (SD)] & $16.4(14.6)$ & $3.49(0.50)$ \\
Patients with IGA score 0-4 [mean (SD)] & $2.7(0.95)$ & 0 \\
0 or 1 & $320(12.0)$ & 0 \\
2 & $610(22.8)$ & $1343(50.2)$ \\
3 & $1288(48.1)$ & $1301(48.6)$ \\
4 & $459(17.1)$ & $30(1.1)$ \\
Missing & 0 & $7.1(1.93)$ \\
Patients with weekly average Pruritus NRS score (0-10) [mean (SD)] & $5.0(2.45)$ & $2528(94.4)$ \\
$\geq 3$ & $1769(66.1)$ & $2437(91.0)$ \\
$\geq 4$ & $1505(56.2)$ & $20.5(5.89)$ \\
POEM total score (0-28) [mean (SD)] & $14.7(8.00)$ & $14.7(7.38)$ \\
DLQI (0-30) [mean (SD)] & $8.5(7.11)$ & \\
\hline
\end{tabular}

Data are expressed as $n(\%)$ unless otherwise specified

$A D$ atopic dermatitis, $B M I$ body mass index, DLQI Dermatology Life Quality Index, EASI Eczema Area and Severity Index, IGA Investigator's Global Assessment, NRS Numerical Rating Scale, OLE open-label extension, POEM Patient-Oriented Eczema Measure, $q 2 w$ every 2 weeks, $q 4 w$ every 4 weeks, $q 8 w$ every 8 weeks, $q w$ weekly, $S D$ standard deviation, $T C I$ topical calcineurin inhibitor, TCS topical corticosteroid

${ }^{a}$ Includes patients from NCT01259323 [14, 19] $(N=7)$; NCT01859988 [20] $(N=296)$; NCT01385657 [14, 19] $(N=12)$; NCT01548404 [14] $(N=51) ; \mathrm{NCT01639040} \mathrm{[14]}(N=17) ; \mathrm{NCT02260986} \mathrm{[10]}(N=581) ; \mathrm{NCT} 01979016$ [18] $(N=44) ; \mathrm{NCT} 02210780[15](N=168)$; NCT02277743 [12] $(N=353) ; \mathrm{NCT02407756} \mathrm{[16]}(N=5)$; NCT02395133 [22] $(N=415) ;$ NCT02277769 [12] $(N=402) ;$ NCT02755649 [11] $(N=313)$; NCT02647086 [17] $(N=13)$

${ }^{\mathrm{b}}$ These patients completed the treatment and end-of-study periods

${ }^{\mathrm{c}}$ Includes reasons of relocation, desire for pregnancy, did not want to discontinue treatment for 12 weeks, work/school conflict, and personal reasons not specified

${ }^{\mathrm{d}}$ Includes patients withdrawn from the study, both those receiving treatment at the time of withdrawal and those not receiving treatment during the safety follow-up period

${ }^{\mathrm{e}}$ Includes the following dupilumab doses in the parent study: $75 \mathrm{mg} \mathrm{qw}, 100 \mathrm{mg} \mathrm{q} 4 \mathrm{w}, 150 \mathrm{mg}$ qw, $200 \mathrm{mg} \mathrm{q} 2 \mathrm{w}, 200 \mathrm{mg}$ qw, $300 \mathrm{mg}$ q8w, $300 \mathrm{mg} \mathrm{q} 4 \mathrm{w}, 2 \mathrm{mg} / \mathrm{kg}, 4 \mathrm{mg} / \mathrm{kg}$

${ }^{\mathrm{f}}$ These patients had screen failed in the parent study because the enrollment target was met, but they were permitted to enter the OLE

headache, oral herpes, and injection-site reactions were the only AEs in $\geq 5 \%$ of patients.

The exposure-adjusted rates of TEAEs and serious TEAEs in this study were consistent with previously reported rates for dupilumab weekly plus TCS (504.5 number of events [nE]/100 PY and $3.7 \mathrm{nE} / 100 \mathrm{PY}$, respectively) and lower than placebo plus TCS (531.9 nE/100 PY and $8.4 \mathrm{nE} / 100 \mathrm{PY}$, respectively) at 52 weeks. Compliance with study treatment was high (98\%) and the rate of treatment discontinuation due to AEs was low (3.5\%). Furthermore, few patients (7.5\%) receiving dupilumab in this trial required use of systemic rescue medication, demonstrating that dupilumab alone or with concomitant TCSs provides longterm disease control in patients with moderate-to-severe AD.

Most conjunctivitis events reported in this study were mild-to-moderate in severity and did not result in treatment discontinuation. The occurrence of conjunctivitis in dupilumab clinical trials has been reported elsewhere [23]. In this analysis, the exposure-adjusted incidence of conjunctivitis (16.14 $\mathrm{nE} / 100 \mathrm{PY})$ was lower than in a previous 52-week controlled trial $(30.60 \mathrm{nE} / 100 \mathrm{PY})$ and in the 76-week analysis of this trial (20.8 nE/100 PY) [13], indicating that event frequency may diminish with continued dupilumab treatment. Similarly, the exposure-adjusted incidence of injection-site reactions (16.70 nE/100 PY) decreased compared with the 52-week controlled trial (78.02 $\mathrm{nE} / 100 \mathrm{PY}$ ) and the 76-week analysis of the OLE (36.5 nE/100 PY), suggesting decreased event frequency over time.

The most common serious TEAEs included squamous cell carcinoma of the skin. Of note, an increased incidence of non-melanoma skin cancers in patients with $\mathrm{AD}$ has been reported in the literature [24-26]. 


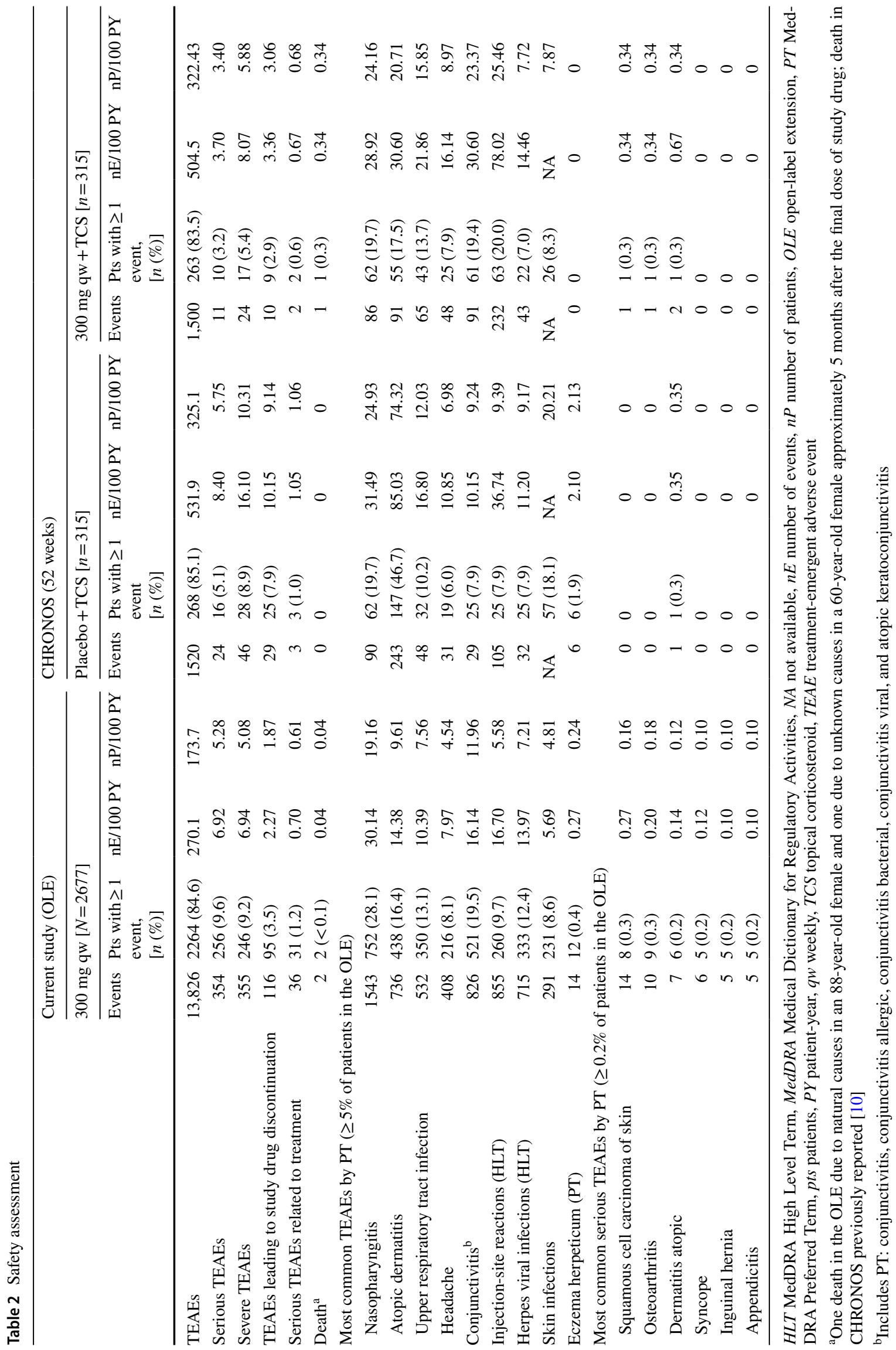


Table 3 Efficacy assessment

\begin{tabular}{|c|c|c|c|}
\hline & \multicolumn{3}{|c|}{ Dupilumab 300 mg qw [ $N=2677]$} \\
\hline & Week $100[n=1028]$ & Week $124[n=529]$ & Week $148^{\mathrm{a}}[n=347]$ \\
\hline Patients achieving IGA 0 or $1[\mathrm{n} / \mathrm{N} 1(\%)]$ & $589 / 1014(58.1)$ & $271 / 458(59.2)$ & 43/58 (74.1) \\
\hline $\begin{array}{l}\text { Patients achieving a reduction in IGA of } \geq 2 \text { from baseline of the parent study } \\
{[n / N 1(\%)]}\end{array}$ & $769 / 996(77.2)$ & $359 / 457(78.6)$ & $58 / 66(87.9)$ \\
\hline Patients achieving EASI-50 [n/N1 (\%)] & $979 / 994(98.5)$ & $445 / 453(98.2)$ & $57 / 58(98.3)$ \\
\hline Patients achieving EASI-75 [n/N1 (\%)] & 908/994 (91.3) & $416 / 453(91.8)$ & $56 / 58(96.6)$ \\
\hline Patients achieving EASI-90 [n/N1 (\%)] & $724 / 994(72.8)$ & $337 / 453(74.4)$ & $51 / 58(87.9)$ \\
\hline EASI (primary analysis), observed values [mean (SD)] & $2.6(3.6)$ & $2.3(3.5)$ & $1.4(3.2)$ \\
\hline OC (sensitivity analysis) [mean (SE)] & $2.6(0.1)$ & $2.3(0.2)$ & $1.5(0.4)$ \\
\hline LOCF (sensitivity analysis) [mean (SE)] & $3.6(0.2)$ & $3.6(0.2)$ & $3.6(0.2)$ \\
\hline MI (sensitivity analysis) [mean (SE)] & $2.9(0.1)$ & $2.8(0.1)$ & $3.5(0.1)$ \\
\hline $\begin{array}{l}\text { Change in EASI from baseline of the parent study, observed values [mean } \\
\text { (SD)] }\end{array}$ & $-31.3(14.1)$ & $-30.0(13.6)$ & $-29.2(14.2)$ \\
\hline LOCF (sensitivity analysis) [mean (SE)] & $-29.8(0.4)$ & $-29.8(0.4)$ & $-29.8(0.4)$ \\
\hline MI (sensitivity analysis) [mean (SE)] & $-30.6(0.4)$ & $-30.7(0.4)$ & $-30.1(0.4)$ \\
\hline Percentage change in EASI from baseline of the parent study, \% [mean (SD)] & $-91.5(12.6)$ & $-92.3(11.0)$ & $-95.4(9.3)$ \\
\hline Weekly Pruritus NRS (primary analysis), observed values [mean (SD)] & $2.3(1.9)$ & $2.3(1.8)$ & $2.2(1.8)$ \\
\hline OC (sensitivity analysis), mean (SE) & $2.3(0.1)$ & $2.3(0.1)$ & $2.1(0.1)$ \\
\hline LOCF (sensitivity analysis), mean (SE) & $2.5(0.1)$ & $2.6(0.1)$ & $2.6(0.1)$ \\
\hline MI (sensitivity analysis), mean (SE) & $2.4(0.1)$ & $2.6(0.1)$ & $2.5(0.1)$ \\
\hline $\begin{array}{l}\text { Change in weekly Pruritus NRS from baseline of the parent study (primary } \\
\text { analysis), observed values [mean (SD)] }\end{array}$ & $-4.7(2.3)$ & $-4.5(2.4)$ & $-4.4(2.4)$ \\
\hline LOCF (sensitivity analysis) [mean (SE)] & $-4.7(0.1)$ & $-4.6(0.1)$ & $-4.6(0.1)$ \\
\hline MI (sensitivity analysis) [mean (SE)] & $-4.8(0.1)$ & $-4.6(0.1)$ & $-4.7(0.1)$ \\
\hline $\begin{array}{l}\text { Percent change in weekly Pruritus NRS from baseline of the parent study, } \\
\% \text { [mean (SD)] }\end{array}$ & $-65.7(30.2)$ & $-64.2(33.4)$ & $-65.4(28.5)$ \\
\hline $\begin{array}{l}\text { Patients achieving } \geq 3 \text { reduction in weekly Pruritus NRS from baseline of the } \\
\text { parent study }[n / N 1(\%)]\end{array}$ & $604 / 764(79.1)$ & $313 / 416(75.2)$ & $168 / 224(75.0)$ \\
\hline Patients achieving weekly average Pruritus NRS $\leq 3[n / N 1(\%)]$ & 686/888 (77.3) & $395 / 510(77.5)$ & $217 / 265(81.9)$ \\
\hline POEM score [mean (SD)] & $5.4(5.1)$ & $5.1(5.3)$ & N/A \\
\hline DLQI [mean (SD)] & $2.9(3.9)$ & $2.9(4.3)$ & N/A \\
\hline
\end{tabular}

$D L Q I$ Dermatology Life Quality Index, EASI Eczema Area and Severity Index, EASI-50 $\geq 50 \%$ reduction in EASI from baseline, EASI-75 $\geq 75 \%$ reduction in EASI from baseline, EASI-90 $\geq 90 \%$ reduction in EASI from baseline, IGA Investigator's Global Assessment, LOCF last observation carried forward, $M I$ multiple imputation, $N 1$ number of patients with non-missing values, NRS Numerical Rating Scale, POEM PatientOriented Eczema Measure, $O C$ observed cohort, $q w$ weekly, $S D$ standard deviation, $S E$ standard error

${ }^{a}$ The relatively low patient numbers at week 148 for IGA and EASI resulted from the temporary removal of these assessments from the end-oftreatment visit with the adoption of Amendment 6 that were restored as of Amendment 7

Dupilumab showed substantial and sustained improvement in $\mathrm{AD}$ signs and symptoms from the baseline of the parent study, with continued incremental improvement beyond 52 weeks of treatment. Sensitivity analyses of EASI and weekly average Pruritus NRS were consistent with analyses of observed values, demonstrating that patient withdrawal, the majority unrelated to treatment, did not influence the results.

In this study, patients received dupilumab $300 \mathrm{mg}$ weekly, different from the currently approved $300 \mathrm{mg}$ every 2 weeks regimen in adults. Controlled studies have demonstrated no differences in safety or efficacy between these regimens [10-12], suggesting that dupilumab
$300 \mathrm{mg}$ every 2 weeks would achieve results similar to those reported here. Of note, the 300-mg weekly dose was originally chosen for this study to increase the likelihood of identifying safety signals and to generate safety data that could adequately support both dose regimens.

The strengths of this study include its size and duration, whereas limitations include the open-label design and lack of a control arm. Furthermore, fewer patients were available at later time points due to timing of this interim analysis, the requirement for patient withdrawal upon regulatory approval of dupilumab in the enrollment country, and the temporary removal of IGA and EASI assessments at week 148. 


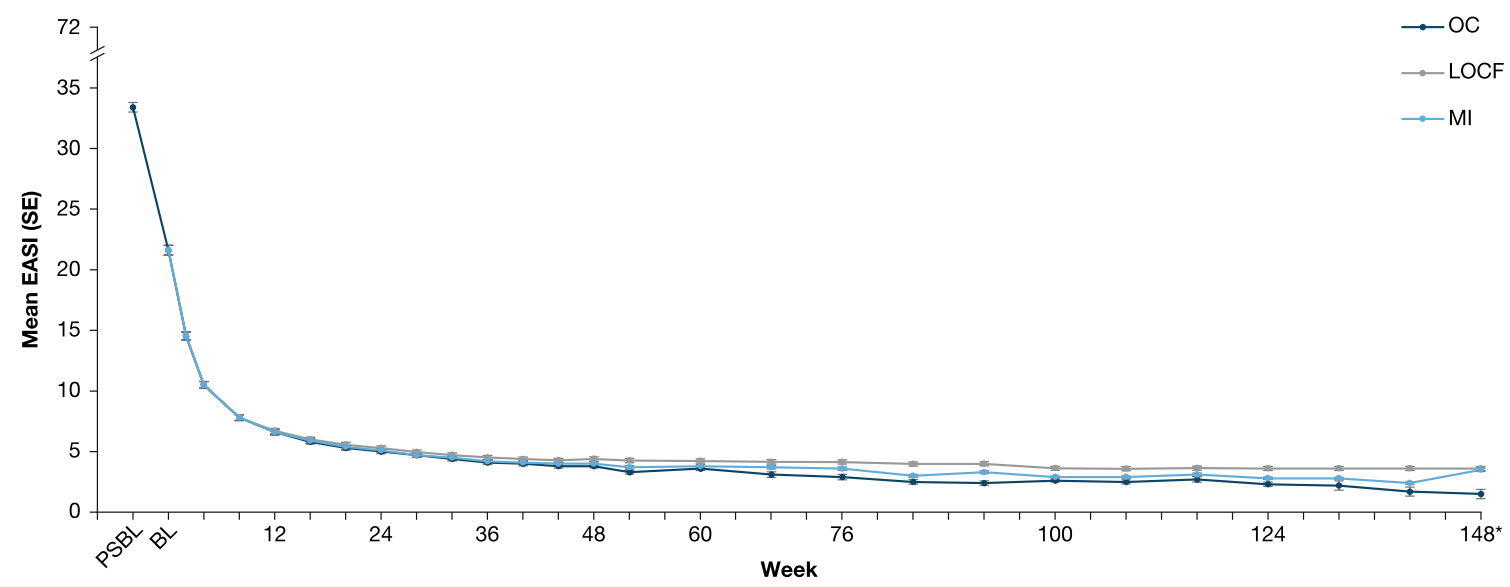

$\begin{array}{rlrrrrrrrrr}\begin{array}{l}\text { No. of patients/ } \\ \text { imputed patients }\end{array} & \text { OC } & 1,3191,343 & 1,310 & 1,265 & 1,232 & 1,210 & 1,189 & 491 & 451 & 898 \\ & \text { LOCF } & 1,343 / 0 & 1,310 / 33 & 1,265 / 78 & 1,232 / 111 & 1,210 / 133 & 1,189 / 154 & 491 / 852 & 86 \\ & \text { MI } & 1,343 / 0 & 1,310 / 33 & 1,265 / 78 & 1,232 / 111 & 1,210 / 133 & 1,189 / 154 & 491 / 852 & 458 & 898 / 445\end{array}$

Fig. 2 Mean EASI over time. *The relatively low patient number at week 148 resulted from the temporary removal of this assessment from the end-of-treatment visit with the adoption of Amendment 6 that was restored as of Amendment 7. BL baseline, EASI Eczema
Area and Severity Index, $L O C F$ last observation carried forward, $M I$ multiple imputation, $O C$ observed cohort, $P S B L$ parent study baseline, $S E$ standard error

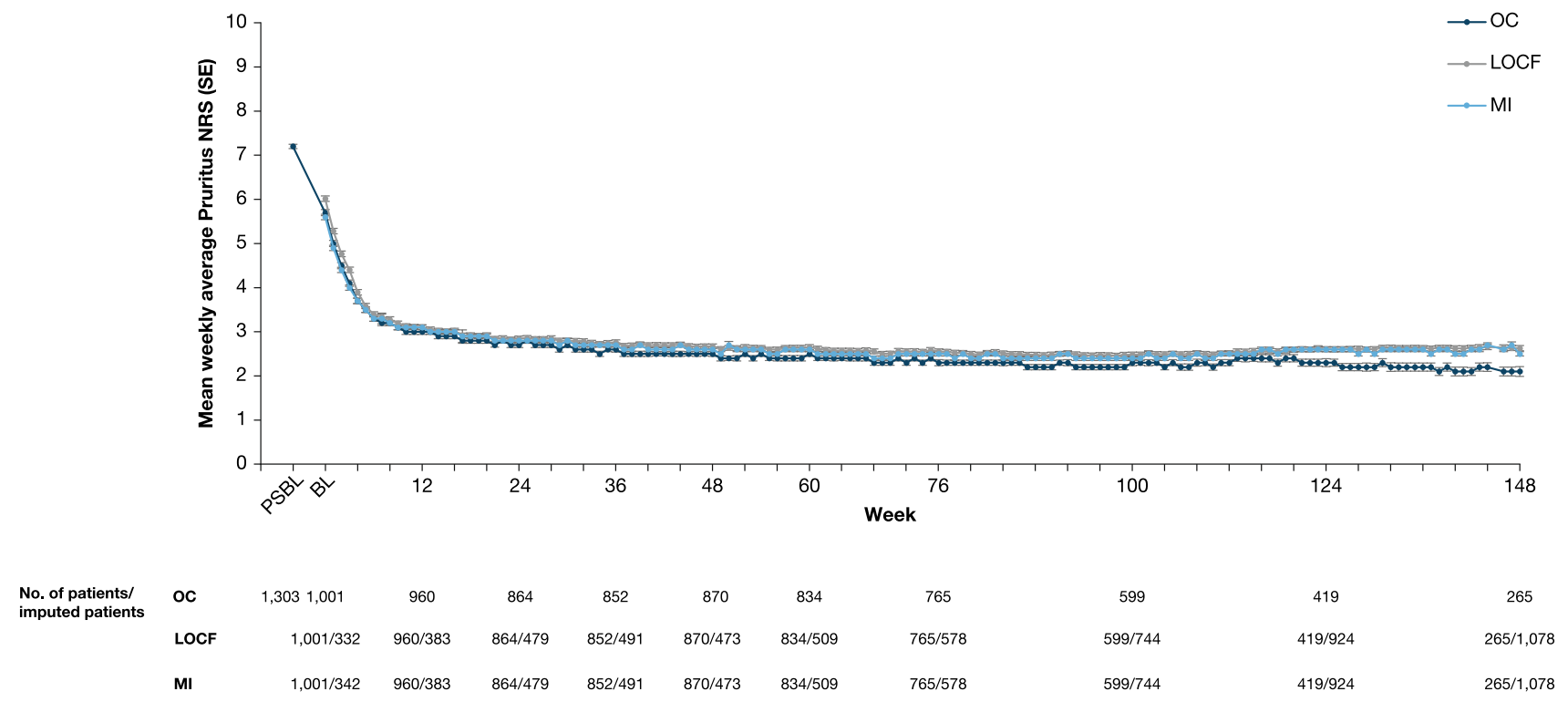

Fig. 3 Mean weekly average Pruritus NRS over time. $B L$ baseline, $L O C F$ last observation carried forward, $M I$ multiple imputation, $N R S$ Numerical Rating Scale, $O C$ observed cohort, $P S B L$ parent study baseline, $S E$ standard error

\section{Conclusions}

The favorable safety and sustained efficacy of dupilumab observed up to 3 years in adults with moderate-to-severe $\mathrm{AD}$ support the long-term continuous use of dupilumab in this chronic, debilitating disease.
Acknowledgements The authors thank the patients who participated in this study, as well as Yufang Lu, MD, Susan Boklage, MS, MPH, and Abhijit Gadkari, PhD, of Regeneron Pharmaceuticals, Inc., Laurent Eckert, PhD, of Sanofi, and Ana B. Rossi, MD, of Sanofi Genzyme for their contributions.

Data Sharing Statement Qualified researchers may request access to study documents (including the clinical study report, study protocol with any amendments, blank case report form, statistical analysis plan) 
that support the methods and findings reported in this manuscript. Individual anonymized participant data will be considered for sharing once the indication has been approved by a regulatory body, if there is legal authority to share the data and there is not a reasonable likelihood of participant re-identification. Submit requests to https://vivli.org/.

\section{Compliance with Ethical Standards}

Funding Open access was funded by Sanofi and Regeneron Pharmaceuticals, Inc. This research was sponsored by Sanofi and Regeneron Pharmaceuticals, Inc. (ClinicalTrials.gov identifier: NCT01949311). The study sponsors participated in the study design; collection, analysis, and interpretation of the data; writing of the report; and the decision to submit the article for publication. Medical writing and editorial assistance was provided by Carolyn Ellenberger, $\mathrm{PhD}$, of Excerpta Medica, funded by Sanofi Genzyme and Regeneron Pharmaceuticals, Inc.

Conflict of interest Lisa A. Beck: AbbVie, LEO Pharma, Pfizer, Regeneron Pharmaceuticals, Inc.-investigator and consultant; Allakos, Arena Pharma, Astra Zeneca, LEO Pharma, Eli Lilly, Novan, Novartis, Pfizer, Regeneron Pharmaceuticals, Inc., Sanofi, UCB, Vimalan-consultant; Medtronics, Pfizer-stock owner. Diamant Thaçi: AbbVie, Almirall, Amgen, Asana Biosciences, Beiersdorf, Boehringer Ingelheim, Celgene, Dermira, DS Biopharma, Eli Lilly, Galapagos, Galderma, GlaxoSmithKline, Janssen-Cilag, LEO Pharma, Merck Sharp \& Dohme, MorphoSys, Novartis, Pfizer, Regeneron Pharmaceuticals, Inc., Sandoz, Sanofi, Sun Pharma, UCB — consultant, advisory board member, and/or investigator. Mette Deleuran: AbbVie, Almirall, Eli Lilly, Galapagos, LEO Pharma, Meda Pharma, Pfizer, Pierre Fabre, Regeneron Pharmaceuticals, Inc., Sanofi Genzyme-received research support and/or honoraria for lecturing, and consulting/advisory board agreements. Andrew Blauvelt: AbbVie, Aclaris, Almirall, Arena, Athenex, Boehringer Ingelheim, Bristol-Myers Squibb, Dermavant, Dermira, Eli Lilly, FLX Bio, Forte, Galderma, Janssen, LEO Pharma, Novartis, Ortho Derm, Pfizer, Regeneron Pharmaceuticals, Inc., Sandoz, Sanofi Genzyme, Sun Pharma, UCB — scientific adviser and clinical study investigator; AbbVie — paid speaker. Robert Bissonnette: AbbVie, Aquinox Pharmaceuticals, Arcutis Antiobix, Asana Biosciences, Astellas, Boehringer Ingelheim, Brickell Biotech, Dermavant, Dermira, Dignity Sciences, Eli Lilly, Galderma, Glenmark, GlaxoSmithKlineStiefel, Hoffman-La Roche, Kiniksa, Incyte, LEO Pharma, NeoKera, Pfizer, Ralexar Therapeutics, Regeneron Pharmaceuticals, Inc., Sanofi Genzyme, Vitae - consultant and/or has received grants/research support; Innovaderm Research—shareholder. Marjolein de Bruin-Weller: Regeneron Pharmaceuticals, Inc., Sanofi Genzyme-Principal Investigator, advisory board member, consultant; AbbVie-Principal Investigator, advisory board member; Pfizer, Eli Lilly, UCB — advisory board member. Michihiro Hide: Kaken Pharmaceutical, Kyorin Pharmaceutical, Kyowa Hakko Kirin, Maruho, MDS, Mitsubishi Tanabe Pharma, Sanofi KK, Taiho Pharma, Teikoku Seiyaku, Torii Pharmaceutical, Uriach-honoraria for lectures. Lawrence Sher: Aimmune, Optinose, Regeneron Pharmaceuticals, Inc., Sanofi Genzyme-advisory board member; Regeneron Pharmaceuticals, Inc., Sanofi Genzyme-speaker fees; Aimmune, Amgen, Astra Zeneca, Circassia, DBV, Galderma, GlaxoSmithKline, Lupin, Merck, Mylan, Novartis, Novo Nordisk, Optinose, Pearl, Pfizer, Pulmagen, Roxane, Sanofi, Spirometrix, Teva, Vectura, Watson-clinical trials funding. Iftikhar Hussain: CSL Behring, Genentech, Optinose, Pfizer-advisory board member; AbbVie, AnaptysBio, Asana Biosciences, Astra Zeneca, CSL Behring, Genentech, GlaxoSmithKline, Gossamer Bio, HAL Allergy, Kiniksa Pharmaceuticals, LEO Pharma, Menlo Therapeutics, Merck, Novartis, Optinose, Pfizer, Regeneron Pharmaceuticals, Inc., Roche, Shire, Vanda-Principal Investigator. Zhen Chen, Faisal A. Khokhar, Bethany
Beazley, Marcella Ruddy, Neil M.H. Graham, Marius Ardeleanu, Brad Shumel: Regeneron Pharmaceuticals, Inc.-employees and shareholders. Naimish Patel: Sanofi-employee, may hold stock and/or stock options in the company.

Ethics approval This study was conducted in accordance with the ethical standards of the responsible committees and the Declaration of Helsinki, and with the International Conference on Harmonisation guidelines for Good Clinical Practice. The trial was overseen by an independent data and safety monitoring board. The protocol was reviewed and approved by Institutional Review Boards/Ethics Committees at all centers.

Consent to participate Written informed consent was obtained from all patients or their proxies.

Open Access This article is licensed under a Creative Commons Attribution-NonCommercial 4.0 International License, which permits any non-commercial use, sharing, adaptation, distribution and reproduction in any medium or format, as long as you give appropriate credit to the original author(s) and the source, provide a link to the Creative Commons licence, and indicate if changes were made. The images or other third party material in this article are included in the article's Creative Commons licence, unless indicated otherwise in a credit line to the material. If material is not included in the article's Creative Commons licence and your intended use is not permitted by statutory regulation or exceeds the permitted use, you will need to obtain permission directly from the copyright holder. To view a copy of this licence, visit http://creativecommons.org/licenses/by-nc/4.0/.

\section{References}

1. Weidinger S, Beck LA, Bieber T, et al. Atopic dermatitis. Nat Rev Dis Primers. 2018;4:1.

2. Gandhi NA, Pirozzi G, Graham NMH. Commonality of the IL-4/ IL-13 pathway in atopic diseases. Expert Rev Clin Immunol. 2017;13:425-37.

3. Boguniewicz M, Alexis AF, Beck LA, et al. Expert perspectives on management of moderate-to-severe atopic dermatitis: a multidisciplinary consensus addressing current and emerging therapies. J Allergy Clin Immunol Pract. 2017;5:1519-31.

4. Macdonald LE, Karow M, Stevens S, et al. Precise and in situ genetic humanization of $6 \mathrm{Mb}$ of mouse immunoglobulin genes. Proc Natl Acad Sci USA. 2014;111:5147-52.

5. Murphy AJ, Macdonald LE, Stevens S, et al. Mice with megabase humanization of their immunoglobulin genes generate antibodies as efficiently as normal mice. Proc Natl Acad Sci USA. 2014;111:5153-8.

6. Hamid Q, Naseer T, Minshall EM, et al. In vivo expression of IL-12 and IL-13 in atopic dermatitis. J Allergy Clin Immunol. 1996;98:225-31.

7. Nomura I, Goleva E, Howell MD, et al. Cytokine milieu of atopic dermatitis, as compared to psoriasis, skin prevents induction of innate immune response genes. J Immunol. 2003;171:3262-9.

8. US Food and Drug Administration. DUPIXENT ${ }^{\circledR}$ (dupilumab). Highlights of prescribing information. https://www.acces sdata.fda.gov/drugsatfda_docs/label/2019/761055s014lbl.pdf. Accessed 27 Mar 2020.

9. European Medicines Agency. DUPIXENT ${ }^{\circledR}$ (dupilumab). Summary of product characteristics. https://ec.europa.eu/health/docum ents/community-register/2019/20190801145601/anx_14560 1_en.pdf. Accessed 27 Mar 2020. 
10. Blauvelt A, de Bruin-Weller M, Gooderham M, et al. Longterm management of moderate-to-severe atopic dermatitis with dupilumab and concomitant topical corticosteroids (LIBERTY AD CHRONOS): a 1-year, randomised, double-blinded, placebocontrolled, phase 3 trial. Lancet. 2017;389:2287-303.

11. de Bruin-Weller M, Thaçi $\mathrm{D}$, Smith $\mathrm{CH}$, et al. Dupilumab with concomitant topical corticosteroid treatment in adults with atopic dermatitis with an inadequate response or intolerance to ciclosporin A or when this treatment is medically inadvisable: a placebo-controlled, randomized phase III clinical trial (LIBERTY AD CAFÉ). Br J Dermatol. 2018;178:1083-101.

12. Simpson EL, Bieber T, Guttman-Yassky E, et al. Two phase 3 trials of dupilumab versus placebo in atopic dermatitis. N Engl $\mathbf{J}$ Med. 2016;375:2335-48.

13. Deleuran M, Thaçi D, Beck LA, et al. Dupilumab shows longterm safety and efficacy in moderate-to-severe atopic dermatitis patients enrolled in a phase 3 open-label extension study. J Am Acad Dermatol. 2020;82:377-88.

14. Beck LA, Thaçi D, Hamilton JD, et al. Dupilumab treatment in adults with moderate-to-severe atopic dermatitis. N Engl J Med. 2014;371:130-9.

15. Blauvelt A, Simpson EL, Tyring SK, et al. Dupilumab does not affect correlates of vaccine-induced immunity: a randomized, placebo-controlled trial in adults with moderate-to-severe atopic dermatitis. J Am Acad Dermatol. 2019;80:158-67.

16. Cork MJ, Thaçi D, Eichenfield LF, et al. Dupilumab in adolescents with uncontrolled moderate-to-severe atopic dermatitis: results from a phase IIa open-label and subsequent phase III open-label extension trial. Br J Dermatol. 2020;182:85-96.

17. Davis JD, Bansal A, Hassman D, et al. Evaluation of potential disease-mediated drug-drug interaction in patients with moderateto-severe atopic dermatitis receiving dupilumab. Clin Pharmacol Ther. 2018;104:1146-54.

18. Guttman-Yassky E, Bissonnette R, Ungar B, et al. Dupilumab progressively improves systemic and cutaneous abnormalities in patients with atopic dermatitis. J Allergy Clin Immunol. 2019;143:155-72.

19. Hamilton JD, Suárez-Fariñas M, Dhingra N, et al. Dupilumab improves the molecular signature in skin of patients with moderate-to-severe atopic dermatitis. J Allergy Clin Immunol. 2014;134:1293-300.

20. Thaçi D, Simpson EL, Beck LA, et al. Efficacy and safety of dupilumab in adults with moderate-to-severe atopic dermatitis inadequately controlled by topical treatments: a randomised, placebo-controlled, dose-ranging phase $2 \mathrm{~b}$ trial. Lancet. 2016;387:40-52.

21. Worm M, Simpson EL, Thaçi D, et al. Efficacy and safety of multiple dupilumab dose regimens after initial successful treatment in patients with atopic dermatitis: a randomized clinical trial. JAMA Dermatol. 2020;156(2):131-43.

22. Vakharia PP, Chopra R, Sacotte R, et al. Severity strata for five patient-reported outcomes in adults with atopic dermatitis. Br J Dermatol. 2018;178:925-30.

23. Akinlade B, Guttman-Yassky E, de Bruin-Weller M, et al. Conjunctivitis in dupilumab clinical trials. Br J Dermatol. 2019;181:459-73.

24. Ruff S, Egeberg A, Andersen YMF, et al. Prevalence of Cancer in Adult Patients with Atopic Dermatitis: a nationwide study. Acta Derm Venereol. 2017;97:1127-9.

25. Cho JM, Davis DMR, Wetter DA, et al. Association between atopic dermatitis and squamous cell carcinoma: a case-control study. Int J Dermatol. 2018;57:313-6.

26. Hagströmer L, Ye W, Nyrén O, Emtestam L. Incidence of cancer among patients with atopic dermatitis. Arch Dermatol. 2005;141:1123-7.

\title{
Affiliations
}

\author{
Lisa A. Beck ${ }^{1}$ - Diamant Thaçi ${ }^{2} \cdot$ Mette Deleuran $^{3} \cdot$ Andrew Blauvelt $^{4} \cdot$ Robert Bissonnette $^{5}$. \\ Marjolein de Bruin-Weller ${ }^{6} \cdot$ Michihiro Hide $^{7}$. Lawrence Sher ${ }^{8}$. Iftikhar Hussain ${ }^{9} \cdot$ Zhen Chen $^{10} \cdot$ Faisal A. Khokhar $^{10}$. \\ Bethany Beazley ${ }^{10} \cdot$ Marcella Ruddy $^{10} \cdot$ Naimish Patel $^{11} \cdot$ Neil M. H. Graham ${ }^{10} \cdot$ Marius Ardeleanu $^{10} \cdot$ Brad Shumel $^{10}$ \\ Brad Shumel \\ brad.shumel@regeneron.com \\ 1 Department of Dermatology, University of Rochester \\ Medical Center, Rochester, NY, USA \\ 2 University of Lübeck, Lübeck, Germany \\ 3 Department of Dermatology, Aarhus University Hospital, \\ Aarhus, Denmark \\ 4 Oregon Medical Research, Portland, OR, USA \\ 7 Department of Dermatology, Hiroshima University, \\ Hiroshima, Japan \\ 8 Peninsula Research Associates, Rolling Hills Estates, CA, \\ USA \\ 9 Vital Prospects Clinical Research Institute, PC, Tulsa, OK, \\ USA \\ 10 Regeneron Pharmaceuticals, Inc., 777 Old Saw Mill River \\ Rd, Tarrytown, NY 10591, USA \\ 11 Sanofi, Bridgewater, NJ, USA
}

5 Innovaderm Research, Montreal, QC, Canada

6 National Expertise Center of Atopic Dermatitis, Department of Dermatology and Allergology, University Medical Center Utrecht, Utrecht, The Netherlands 